\title{
Clinical Manifestations of Candidiasis: Beyond Candida albicans
}

\author{
Courtney Smith and Michael C Hanna* \\ Department of Biology, University of Texas at San Antonio, USA
}

*Corresponding author: Michael C Hanna, University of Texas at San Antonio, San

Antonio, USA, Email: michael.hanna@utsa.edu

\section{Review Article}

Volume 3 Issue 3

Received Date: July 18, 2019

Published Date: August 23, 2019

DOI: $10.23880 /$ vij-16000219

\section{Abstract}

Fungal infections, including the Candida, species, are increasingly becoming problematic over the last two decades particularly in immunocompromised individuals. While there are over 150 different species only a few are pathogenic. The Candida species can evade the immune system and become pathogenic by a variety of factors including morphology, escaping phagocytosis, expression of adhesins and invasins, biofilm production due to contact sensing and thigmotropism, inhibition of the cytokine, interleukin 17, and production by the host. Candida infections produce both non-life, and life threatening manifestations and certain risk factors make infections more likely. Clinical manifestations include oral, genitourinary, gastrointestinal, and cutaneous candidiasis. Diagnosis of candidiasis can be problematic, however antigen testing, agar growth, and microscopic examination are available. Treatment of candidiasis rely on three principal antifungal agents; azoles, polyenes, and echinocandins. These three drug classes have specific drug target.

Keywords: Pseudohyphae; Chlamydospores; Hyphal Cells; Albicans

\section{Abbreviations: PAMPs: Pathogen-Associated} Molecular Patterns; TLRs: Toll-like Receptors; CLRs: Ctype Lectin Receptors; MR: Mannose Receptor.

\section{Introduction}

The occurrence and prevalence of invasive fungal infections have been increasing over the past few decades, particularly in the immunocompromised patients and or individuals with predisposing conditions [1]. The increase of fungal infections over the past two decades is multifactorial, including patient demographics, higher occurrence of chronic diseases, and immunocompromised individuals, etcetera [2].

Fungal pathogens can be broadly grouped into three categories: opportunistic, true pathogens, and rare "emerging" opportunistic fungi. The difference between a true and opportunistic pathogen is that a true pathogen is an infectious agent that causes disease in any susceptible host. In comparison, an opportunistic pathogen is a causative agent that generally only causes disease in the immunocompromised individuals and rarely in healthy patients. Lastly, rare "emerging" opportunistic fungi are pathogens that are not commonly seen and are generally reported in severely immunocompromised patients.

Fungal species generally grow in three main morphologies: yeast, hyphae, and pseudohyphae. However, Candida species have been noted to also produce chlamydospores in vitro and rarely in infected tissues. Yeast cells are single cells that are spherical and can display both axial and bipolar budding patterns. Hyphae and pseudohyphae are also referred to as filamentous morphologies because the cells generally grow in a polarized manner and elongate. What 


\section{Virology \& Immunology Journal}

differentiates these two cells types is that hyphae cells are uniform in width and have true septa lacking constrictions, whereas pseudohyphae are ellipsoidal and have constrictions at the septal junctions. In both yeast and pseudohyphae, nuclear division occurs, and septum ring forms entirely across the mother-daughter bud neck. On the other hand, those events occur within the germ tube during the first cell division for hyphal cells. Additionally, the initial germ tube development happens before the G $1 / \mathrm{S}$ phase transition, whereas in yeast and pseudohyphae, in synchrony with the cell cycle. Upon finishing the first cell division, hyphal cells arrest in G1 cell phase until enough cytoplasmic mass has accumulated to move into the next cell cycle. As a result, hyphal cells have less branched compared to pseudohyphae cells. All these morphologies discussed are vegetative cell types. The final morphological structure is the chlamydospores. These are large, round, and thickwalled cells that customarily formed at the ends of hyphae filaments. Chlamydospores tend to form under stressful and poor nutrient environments. Two Candida species have been cited as forming chlamydospores: $C$. albicans and C. dubliniensis, along with other classical dimorphic fungi $[3,4]$. This cell structure is rare, and little is known about its function.

The Candida species are members of the kingdom Fungi, the phylum Ascomycota, class Hemiascomycota, and lastly the order Saccharomycetales [5,6]. There are more than 150 known Candida species; however, only 15 of those species are of clinical importance: Candida albicans, Candida glabrata, Candida tropicalis, Candida parapsilosis, Candida krusei, Candida guilliermondii, Candida lusitaniae, Candida dubliniensis, Candida pellicu-losa, Candida kefyr, Candida lipolytica, Candida famata, Candida inconspicua, Candida rugosa, and Candida norvegensis [7]. Isolation frequencies vary among these species, but it has been determined that approximately $95 \%$ of Candida infections are caused by C. albicans, $C$. tropicalis, C. glabrata, C. parapsilosis, and C. krusei [7]. Candida species are either diploid or haploid. A few Candida species that are haploid include C. glabrata, $C$. lusitaniae, and C. guilliermondii [8,9]. The other Candida species including C. tropicalis, C. parapsilosis, C. albicans, and C. dubliniensis are all diploid [8]. However, C. albicans has been seen in the diploid state; this state has been observed during periods of increased stress and during mating.

Candida albicans natural isolates are diploid and initially thought that it did not to have a sexual form of reproduction. While conventional meiosis has not been observed; C. albicans has two mating types (a and alpha).
These a and alpha stains have been shown to be capable of mating and form a tetraploid. This alternation of the cell type from diploid to tetraploid and then return to diploid without meiosis is termed 'parasexual.' Before undergoing the parasexual cycle, diploid cells must switch to an opaque phase. Once in the opaque phase, mating is like Saccharomyces cerevisiae.

Candida albicans is one of the most researched and clinically isolated opportunistic pathogens. Non-albicans Candida species (NAC) has been on the rise of being reported as colonizers and causative agents [5]. Many of these NAC infections account for approximately $50 \%$ of the non-superficial Candida infections; most commonly caused by C. glabrata, C. tropicalis, and C. parapsilosis $[5,10]$.

While specific aspects of Candida species have been discussed in previously published papers; they primarily focused on Candida albicans. This review focuses on complying with current knowledge of epidemiology and pathogenicity, clinical manifestations and therapeutic strategies beyond Candida albicans that are of clinical relevance.

\section{Candida Species Distribution}

There are over 150 species of Candida in nature, but only a small portion are human pathogens. Over the past few decades, the incidence of $C$. albicans has decreased and has begun to shift to non-albicans species. This shift has many factors and may be due to immunosuppression, increase the use of broad-spectrum antibiotics, and an increase in older infected patients. As the Candida infections shift more to non-albicans species, the concern increases.

In North America, C. albicans was isolated 64\% in Canada and 48.9\% I in the USA in during the 1990's but has since decreased to $38 \%$ over the past two decades and shifted towards C. tropicalis, C. glabrata, and C. parapsilosis [7]. Upon looking at Candida species causing invasive candidiasis, in Latin America C. albicans was isolated most often followed by C. parapsilosis. However, when compared to North America, C. glabrata was more common than in Latin America [7]. These results are assumed to be associated with poor maintenance of indwelling medical devices and infection control methods.

In European countries, the distribution of Candida species varies from country to country. For instance, in Finland C. albicans was found to be the most common species followed by C. glabrata; this distribution is similar to that of Sweden and Switzerland [7]. Whereas, in Spain 


\section{Virology \& Immunology Journal}

C. albicans was isolated less than half followed by $C$. parapsilosis being the most common non-albicans species. This distribution was like that of Italy. Other European countries, including Germany, Denmark, and the United Kingdom, C. albicans was isolated the majority of times followed by C. glabrata [7].

Until recently, Candida albicans was the predominant species worldwide causing at least $80 \%$ of the reported Candida infections. Nevertheless, over the past years, previous studies have shown a shift in the epidemiology from Candida albicans to non- albicans Candida which include C. kruesi, C. glabrata, and C. tropicalis $[11,12]$. This shift may be due to immunosuppression, increase the use of broad-spectrum antibiotics, and an increase in infection of older patients. As the Candida infections shift more to non-albicans species, the concern increases. With the emergence of non- albicans Candida infection introduces antifungal resistance. $C$. tropicalis and $C$. parapsilosis are ordinarily susceptible to azole treatment; however, C. tropicalis is less vulnerable to fluconazole as compared to its more pathogenetic counterpart, $C$. albicans. Furthermore, C. glabrata is inherently more resistant to antifungal treatments, especially to fluconazole. C. krusei is naturally unaffected by fluconazole therapies. Lastly, C. lusitaniae is ordinarily able to be treated with azoles, but this species has a higher occurrence of amphotericin $B$ resistance [1].

\section{Pathogenicity of Candida Species}

Candida albicans remains to be the most common clinical isolate causative agent of candidiasis [13]. In most individuals, Candida is a harmless commensal. However, under certain conditions, Candida causes a wide range of infections. Candida pathogenetic potential is multifactorial. Below the pathogenetic mechanisms of Candida and host immune response are discussed.

\section{Virulence Factors}

\section{Morphology}

The yeast form of Candida is not necessary for virulence, but this morphology is known to play essential roles in virulence-related processes including colonization, rapid dissemination, cell-surface adhesion, and biofilm formation [4]. Yeast cells are thought to play a primary role in dissemination; whereas hyphal cells are primarily involved more in invasive infections. Candida will switch to pseudohyphae form to increase nutrition scavenging; in hyphal form serves to invade host tissues, lysis macrophages and neutrophils [4]. Seven clinically relevant Candida species have been documented forming pseudohyphae (C. glabrate, C.lusitaniae, C. guilliermondii,
C. parapsilosis, C. tropicalis, C. dubliniensis, and C. albicans); also only three species have been noted forming the main morphologies (C. albicans, C. tropicalis, C. dublininensis) [4]. The ability to switch between various morphologies aids in adaptation and persistence in different niches inside a host.

Asteroid bodies (AB) have been described in Candida; these bodies are responsible for invasive mycosis [14]. The morphology of an $\mathrm{AB}$ is depicted as concentric layers and molded around a central yeast [14]. The AB protects the yeast cell from the host immune system and the surrounding environment. This structure is considered as an essential morphology because the central yeast is viable and capable of filamenting [14].

\section{Escaping Phagocytes}

C. tropialcis, C. krusei, C. parapsilosis, and C. guilliermondii are efficiently phagocytosed. However, these species filament inside and outside of macrophages to evade phagocytosis and alter macrophage recruitment [15]. Candida species unable to undergo dimorphic transitions have developed strategies to evade phagocytosis. For example, $C$. lusitaniae escapes macrophages by constructing cell chains, and C. glabrata proliferates within macrophages causing the host cell to lysis [16,17].

C. albicans has evolved strategies to allows survival by manipulating and escaping phagocytic cells. C. albicans promotes neutralization of the phagolysosome; this process involves the Stp2 transcription factor induction of the Ato5-mediated ammonia exporter [18]. This neutralization permits hyphae formation, therefore, facilitating escape from macrophages.

Candida species also evade phagocytosis by shielding the surface PAMPs. For the host to phagocytose pathogens, the pathogen must first be sensed; this process of sensing is achieved through recognizing the PAMPs of Candida. One study showed that the Candida $\beta$-glucans were shielded from Dectin-1 by external call well components [19]. The study also noted that during yeast budding and cell separation the $\beta$-glucans were not protected; whereas, during filament growth, the $\beta$-glucans fail to activate Dectin-1. As a result, the morphology directly contributes to the method by which phagocytes recognize the pathogen [19].

\section{Adhesins and Invasins}

Candida species have a set of proteins which mediates adherence between Candida cells to other materials, which includes microorganisms, surfaces, other fungal 


\section{Virology \& Immunology Journal}

cells and host cells. Agglutinin-like sequence (ALS) proteins have eight members (ALS1-7 and ALS9). The ALS gene encodes for glycosylphosphatidylinositol (GPI)linked cell surface glycoproteins; these are located on the surface or wall of C. albicans [20]. Of the eight ALS proteins, ALS3 is vital for adherence of C. albicans in mucosal membranes including oral and vaginal [20]. ALS3 is hyphae specific and is required for endocytosis; also, ALS3 binds to host cell surface proteins $\mathrm{N}$-cadherin on endothelial cells and E-cadherin on the oral epithelial cells.

Other crucial adhesins are HWP1 which is a hyphaeassociated GPI-linked protein [20]. This protein is only expressed in the filamentous form. HWP1 acts as a substrate of mammalian transglutaminases in the epithelial cells which causes covalent bonding between host and fungi. HWP1 $\Delta / \Delta$ mutants showed a decrease in adherence to buccal epithelial cells and reduced capabilities in causing systemic candidiasis [21].

Both ALS3 and HWP1 play an important and essential role in biofilm formation.

\section{Contact Sensing and Thigmotropism}

Candida has evolved to have an environmental cue that triggers hyphae and biofilm production. Upon encountering a surface, the Candida yeast cells will switch and begin to grow in hyphae form. When on a surface with ridges hyphae with grow directionally. This process is called thigmotropism. Thigmotropism begins when the cell encounters a ridge and causes the membrane to stretch which is detected by Mid1p. Followed by extracellular calcium uptake; this influx redirects the cell polarity machinery to a new growth axis. Evidence has been provided that thigmotropism is required for the invasion and damage of epithelial cells [22]. Consequently, contact sensing is essential for pathogenicity.

\section{Biofilm Formation}

Multicellular communities composed primarily of a polysaccharide matrix that adhere and grow on the surface of tissues or indwelling medical devices are termed biofilms [23-25]. Cellular biofilms exhibit drug resistance because of the heterogeneity of morphologies and the fungal metabolic state. A known property that contributes to drug resistance is the polysaccharide $\beta-1$, 3 -glucans. The biofilms extracellular matrix can sequester some antifungal drugs reducing the drug's effectiveness. The resistance is due to the up regulation of two efflux pumps: ATP-binding cassette $(\mathrm{ABC})$ transporter and the major facilitator superfamily (MFS) [26]. ABC transporters are a multigene family, including CDR genes, that plays a direct role in resistance; MFS has the MDR1 gene that encodes for a significant facilitator that has been linked to azole resistance and when overexpressed leads to fluconazole resistance entirely [27]. Biofilms also have a communication network that is composed of signaling processes, which includes quorum sensing [23]. This sensing process responses directly the cell density that affects growth and morphological changes; this most likely aids in survival [23]. Biofilm formation can be broadly categorized into three phases: adherence of yeast to a surface, the formation of a yeast-hyphae matrix, and an increase of matrix material [28]. Fully matured Candida biofilms have a complex network of yeast, hyphae, and pseudohyphae in a polysaccharide, carbohydrate, and protein environment. Following maturation yeast cells may disperse from the biofilm to seed at new, distal sites. The development of a biofilm is multifactorial. The chemical makeup of the surface has been documented to influence biofilm formation; also, high glucose mediums aid in biofilm formation [29]. Additionally, another study showed cell surface hydrophobicity, and gentle shaking promotes biofilm development [30].

Most Candida species have been shown to form a biofilm under various temperatures in vitro [28]. One study created a model system to investigate the growth of Candida biofilm on catheter material as determined by dry-weight, colorimetric, or radioisotopes assay. They showed three Candida species (C. glabrata, C. parapsilosis, and $C$. pseudotropicalsis) grew significantly less biofilm compared to their more pathogenetic counterpart, $C$. albicans [29]. Another study confirmed these findings by using similar methods and determined that C. albicans form a more complex biofilm in comparison to $C$. parapsilosis, in which this biofilm consisted of only blastospore clumps [31]. Additionally, the C. albicans isolates showed minimal variation in biofilm production; also, C. parapsilosis, C. tropicalsis, and C. glabrata showed less biofilm development [31]. Candida species vary in biofilm production capabilities. This highlights the importance of evaluating biofilms and Candida species wild phenotypes, both of which may contribute to pathogenicity.

\section{Inhibition of IL-17 production}

Interleukin 17 (IL-17) has been demonstrated to have an essential role in the host defense against fungal infections. A recent study showed that Candida could directly inhibit -17 production by altering the host tryptophan metabolism [32]. Tryptophan metabolism is monitored by two enzymes: indolamine-2,3-dioxygenase 
(IDO) and tryptophan hydroxylase. C. albicans can shift tryptophan metabolism by inhibiting IDO expression, resulting in a reduced number of kynurenines and an increase in 5-hydroxytryptophan metabolites. The increase in the metabolites consequently inhibits the host production of IL-17 [32].

\section{Farnesol}

Farnesol was identified as a quorum-sensing molecule that represses yeast-hyphae transition of $C$. albicans [33,34]. It has been determined that farnesol may decrease macrophage function through ROS production. Additionally, farnesol has been suggested as a protective factor for Candida against oxidative stress by upregulating gene expression of CAT1, SOD1, SOD2, and SOD4 [33].

On the other, Candida has been cited to block farnesol production. A study investigated C. albicans mating; the results showed that farnesol blocks aerobic mating because morphological switching from yeast to opaque cells is required for mating [34]. They also determined that farnesol kill the opaque cells when under aerobic conditions. Also, under anaerobic conditions, the opaque cells were stabilized and blocked the production of farnesol, which permitted mating [34]. These findings suggest that anaerobic sites, for example, the gastrointestinal tract, are natural niches for ongoing colonization and mating for $C$. albicans.

\section{Host response to Candida infections?}

The fundamental aim of the host innate immune system is to distinguish between self and not self. Host recognition of invading microorganisms relies on evolutionarily conserved, germline-encoded patternrecognition receptors (PRRs), which recognize highly conserved invariable molecular patterns, often essential for the survival of microorganisms, called pathogenassociated molecular patterns ( PAMPs). Candida cell wall is composed of chitin, $\beta$-glucans, and mannoproteins [33]. These cell wall structures are recognized by two classes of membrane-bound PRRs: Toll-like receptors (TLRs) and Ctype lectin receptors (CLRs). TLRs are transmembrane receptors; their engagement and induction send a signal to the nucleus of a cell causing gene expression of cytokines and chemokines. A major TLR that recognizes $C$. albicans is TRL2 which detects phospholipomannan; also TLR4 recognizes 0-linked mannan [33]. Furthermore, by being able to recognize membrane-bound PAMPs, several PRRs identify Candida intracellularly. TLR9 has been shown to recognize Candida DNA and encourage cytokine production within dendritic cells [35]. Another study investigated how TRL9 modulated macrophage antifungal effector function. The results demonstrated the TLR9

\section{Virology \& Immunology Journal}

deficiency was linked to resistance to murine candidiasis [36]. Macrophages lacking TLR9 were described to have normal phagosomal maturation; macrophage tumor necrosis factor alpha (TNF- $\alpha$ ) was also increased when the TLR9 deficiency was present [36]. Macrophage effector response was increased by nitric oxide production. Moreover, TLR9 knockout had a more effective microbicidal activity against C. albicans [36]. An adaptor molecule termed MyD88-dependent of the TLR pathway is required for Candida and other fungal resistance; MyD88 signals through IL-1R, TLR2, TLR4, and TLR9.

A second major PRR family that identifies Candida PAMPs is the CLRs. There multiple PRRs associated with the CLRs family including Dectin-1, Dectin-2, Dectin-3, DC-SIGN, mannose receptor (MR) and mincle. $\beta$-glucans are identified by Dectin-1 and signals through Syk; Nlinked mannan is recognized by mannose receptors. Dectin-2 and Dectin-3 bind to mannans and signals through Syk; DC-SIGN is found on dendric cells and binds to high mannose carbohydrates. MR binds terminal mannan, and mincle binds $\alpha$-mannose. In the Syk signaling pathway, CARD9 is signaled and induces cytokine induction. The current understanding of CLRs role in host defense is limited to C. albicans. One study investigated the potential role of Dectin- 1 and Dectin- 2 in host defense against $C$. glabrata. The study yielded results of the animal model becoming more susceptible to infection [37]. Dectin-1 was determined to be mandatory for myeloid cell recognition, killing of C. glabrata, and the development of Th1 and Th17 cell-mediated adaptive immune responses. Also, in Dectin- 1 deficient mice, the host inflammatory responses were impaired. In conclusion, Dectin-1 plays a significant role in the host immune response against Candida pathogens [37].

(NOD)-like receptors (NLRs) are another PRRs family that recognize fungal PAMPs. NLRs are involved in recognize recognizing intracellular pathogens. To date, there are 22 known NLRs, but only three have been seen to play a role in fungal recognition, this includes NLRP3, NLRP10, and NLRC4 [38,39]. NLRP3 is important for $C$. albicans induced inflammation; which is consistent because of NLRP3 signals through inflammasome formation and aids in the generation of Th17 responses [38,39]. Additionally, NLRP10 and NLRC4 have both been related to anti-C. albicans responses [40,41]. A study showed that when NLRP10 was deficient, there was an increase in susceptibility for disseminated candidiasis, no alternation in proinflammatory cytokine production, and detective Th1 and Th17 responses [40]. These results show the novel role of NLRP10 in the context of adaptive 


\section{Virology \& Immunology Journal}

fungal immune responses. In other respects, NLRC4 was determined to function within the mucosal stroma to control oral candidiasis; also, NLRC4 impacts inflammatory cell recruitment and protect against disseminated infections [41]. In conclusion, this study revealed the specific role of the NLRC4 inflammasome in innate Candida immune responses.

\section{Clinical Manifestations and Risk Factors}

Candida species present as a commensal and an opportunistic pathogen; it is the most common cause of fungal infections [42]. These fungal infections range from non-life threatening mucocutaneous disease to invasive, life-threatening infections [43]. Candida infections commonly affect immunocompromised patients. Many factors affect clinical manifestations of candidiasis; also, there are many clinical presentations of candidiasis.

\section{Risk factors for candidiasis}

Even though Candida species are resident organisms of the human microflora, in the past couple of decades, abnormal overgrowth of Candida has been observed. This overgrowth is of importance because it has been noted to be the direct cause of a wide range of symptoms in healthy individuals [44]. Numerous factors contribute to yeast infections. These predisposing conditions include, but not limited to, decreased digestive secretions, nutritional deficiencies and dietary factors, impaired immune system, impaired liver function, drug and prolonged antibiotics use [7,44]. Risk factors for candidiasis can be divided into two general groups: host or health-care related factors. Immunosuppressive diseases, neutropenia, and age are the predominant hostrelated factors that are host-related that increases susceptibility to candidiasis [7]. Prolonged hospital or ICU visits are the most common health-care associated risk factor [45].

\section{Altered Digestive Factors and Other Factors}

In a healthy individual, digestive secretion directly contribute $s$ to a healthy gut flora which includes preventing Candida overgrowth and its infiltration into the GI tracts surfaces. Pancreatic enzymes are responsible for maintaining the integrity of the small intestine; dysfunction of these enzymes can lead to food allergies and or formation of toxic substances [44]. A recent study investigated the prevalence of oral candidiasis and its association with malnutrition. The study finds determined that oral Candidal infections appeared to be related to malnutrition; therefore, mucosal lesions have a negative impact on the patient's nutrition and overall health [46]. Consequently, restoring and maintaining proper digestive secretions is of high importance to combat Candida.
All organisms need a balanced diet of right proportions; when that balance becomes unbalanced, the well-being of the body becomes compromised. Certain foods in our daily diet may favor Candida growth, for example, cheeses, alcoholic drinks, and milk; all these foods contain a higher content of yeast. Dairy products contain a high amount of lactose which may stimulate fungal growth, but they also have antibiotics [44]. Therefore, dietary factors should be monitored to ensure there is a balance. Also, common nutrient deficiencies in patients with chronic candidiasis include zinc, magnesium, selenium, fatty acids, folic acid, and vitamins B6 and A [44]. Also, iron is now considered an essential micronutrient. Multiple recent studies have documented irons role in fostering and colonization of Candida and other yeast $[47,48]$.

Another factor is impaired liver function. When the livers functions are modified, toxins begin to accumulate. The accumulation can result in liver damage, changes in the mechanisms of detoxification, which in turn allows Candida to overgrow and can cause chronic candidiasis of the GI tract $[44,49]$. Maintaining the microflora balance is vital to keep the growth of Candida in check.

\section{Immunocompromised patients}

There are a plethora of mechanisms that are designed to protect the host from fungal pathogens; this protection is dependent upon an amalgamation of factors. Within immunocompromised individuals, alternations in phagocytic cells presence or function are the most common risk factor that puts the patients at a higher risk of fungal infections [50]. Maintaining the proper conditions for an appropriate immune function is critical to prevent infections from microorganisms and opportunistic pathogens. Therefore, any disease that affects the immune system function increases susceptibility infection. The most common predisposing diseases are diabetes, cancer, HIV/AIDS, and neutropenia [44].

\section{Diabetes and Candidiasis}

Diabetes is a prevalent disorder that is characterized by insufficient insulin secretion or insulin resistance to metabolic action on a target tissue in both men and women [49]. Oral candidiasis is common among diabetic patients; it has been shown that elevated blood glucose levels favor Candida growth but the mechanism which predisposes these patients is unclear [50]. The frequency of Candida infections among people with diabetes is approximately 31\%; diabetic patients also have an increase in Candida colonization of the oral and vaginal cavity when compared to healthy patients $[49,51,52]$. 


\section{Virology \& Immunology Journal}

Studies have shown that women with type 1 diabetes are more likely to be colonized with Candida species as compared to type 2 patients [51]. Female diabetic patients were commonly colonized in the vaginal cavity by either $C$. albicans or C. glabrata [11,51]. Patients with type 1 diabetes were predominately colonized by $C$. albicans whereas women with type 2 C. glabrata was more common [51].

\section{HIV/AIDS and Candidiasis}

The most common Candidal infection among the HIV/AIDS population is oropharyngeal [50]. C. albicans is the prevailing strain among these patients (68.5\%) $[49,50]$, followed by C. tropicalis $(7.4 \%)$, C. krusei $(6.4 \%)$, C. parapsilosis $(3.0 \%)$, and lastly C. sake $(2.5 \%)[49,53]$. HIV/AIDS patients have an increase in risk for oral candidiasis due to the decreased function of Th17; these T helper cells are pivotal in guarding against Candida [49]. Because of this loss and the progression of HIV, this allows the fungus to no longer be contained by the oral mucosa, subsequently allowing C. albicans to become pathogenic $[49,50]$.

A recent study has shown that $C$. albicans could inhibit the replication of HIV-1 within macrophages and dendritic cells $[49,54]$. This inhibition does not act alone; host restriction factors also contribute; primarily by stimulating interferon- $\alpha$ and restriction factors APOBEC3G and APOBEC3F production [54]. These two restriction factors introduce $G$ to $A$ substitutions of the HIV-1 genome resulting in viral replication inhibition $[49,54]$. Even though, Candida infection silences HIV-1 this could be problematic because this mechanism may help HIV-1 to evade the host immune responses and promote antiretroviral resistance [54].

Previous studies have shown there is a significant correlation between Candida colonization and CD4+ lymphocytes. These studies demonstrated a decreased count of CD4+ T lymphocytes is commonly correlated with the increased frequency of oral candidiasis [55-57]. $\mathrm{T}$ helper cell 17 has also been noted to become depleted as the HIV infection progresses resulting in C. albicans to overgrow and overcome the epithelial defenses [58]. However, new insights are needed to combat Candida and other fungal infections under immunocompromised conditions.

\section{Cancer and Candidiasis}

Cancer and chemotherapy predispose patients to opportunistic fungal infections because of the individual's immunosuppression. Altered immunological factors correlate with increased susceptibility to Candida infections within cancer patients. Chemotherapy directly causes mucosal damage and impairs the cellular and humoral immune responses. This route of treatment tends to alter the host immune response. For example, a study showed that cancer patients had a deficiency in myeloperoxidase (MPO) and were at an increased risk for Candida infections [59]. More times than not Candidal infections are a common side effect of chemotherapy against malignant cancers. Another study determined that thrombospondin-1 enhance Candida ability to disseminate due to the imbalance of the host immune system; this results in decreased fungal clearance, dissemination, and increased mortality [60].

Studies have shown that patients with candidiasis may have an increase in developing cancer later in life. OIn one study conducted a nationwide population-based cohort study to evaluate whether Candida infections is are related to increased cancer risk. The study concluded that patients with Candida infections had a significantly higher risk of cancer overall [61]. Other recent findings have reported that $C$. albicans is capable of promoting cancer through various mechanisms: producing carcinogenic byproducts, promoting inflammation, and induction of Th17 response [62].

Currently, there is insufficient data that supports Candida species can promote cancer. However, there is an acceptable hypothesis about the carcinogenic effects of Candida species. Studies published years ago implied that C. albicans might contribute to oral carcinogenesis because of its ability to produce nitrosamines $[63,64]$. As a result, carcinogenic lesions may develop because of the activation of specific proto-oncogenes [63]. These findings were supported by another research study that concluded that C. albicans acted as a promoter for carcinogenesis on rat tongues followed by repeated application of nitroquinoline [65]. Besides, a more recent study showed that $C$. albicans also produces high levels of acetaldehyde in low oxygen conditions [66]. These findings are of importance because acetaldehyde is carcinogenic and has been associated with cancers of the upper digestive tract [66]. Better More insights are needed in this field to obtain a better understanding of the carcinogenic byproducts Candida produces and its downstream effects.

Inflammation and cancer have had a recognized relationship for years. Inflammation is necessary for the progression of cancer and the malignant phenotype. Candidal infections are responsible for pro-inflammatory cytokines to be secreted. The effects of this fungal 


\section{Virology \& Immunology Journal}

infection to endorse metastasis appears to be based on inflammation [62]. It has been noted experimentally that following the recognition of Candida contributes to tumor adhesion and metastasis in correlation with TNF- $\alpha$ and IL$18[67,68]$. This is critical because patients are commonly treated with chemotherapy and are immunosuppressed meaning their leukocyte population is substantially reduced or eliminated. As a result, cancer cells may not be adhering to the leukocytes but instead attaching to endothelium and consequently metastasizing.

Another mechanism proposes that $C$. albicans may promote cancer progression. Th17 produces IL-17 which is a known requirement for resistance against Candida. However, other cytokines like IL-23 are also involved in angiogenesis, tumor incidence, and growth [69]. IL-23 antagonizes IL-12 and IFN- $\gamma$; both are required cytokines for a cytotoxic immune response. When IL-23 is being expressed within a tumor environment, IL-17 can be induced by tumor resident immune cells. The IL-17 expression induces neutrophils and macrophage infiltration [69]. Consequently, these cells produce matrix metalloproteinase (MMPs) which endorses tumor growth [69]. Additionally, IL-23 might induce cytotoxic T lymphocytes (CTLs) which produce IL-17 resulting in tumor progression rather than tumor elimination [69]. Although the roles of IL-23 and IL-17 are still being investigated, inappropriate expression of these cytokines has been linked to cancer.

There are numerous studies reporting mechanisms by which viruses and bacteria can impact cancer directly, but there is limited research in the context of fungi. To obtain a vast knowledge and to develop appropriate treatments more research is needed to understand better how Candida species promotes cancer.

\section{Prolonged use of Antibiotics and Drugs}

Antibiotics have been determined to play a significant role in the development of Candida infections [44]. Overgrowth of Candida occurs because the mode of action for antibiotics is to eliminate bacteria; which has the role of preventing the overgrowth of fungal species [44]. $C$. albicans is the primary species that overgrows $[44,50]$. Antibiotics with a narrow spectrum are less likely to cause fungal overgrowth due to these therapies are more specific in their mode of killing. The fact that fungal growth increases with the broad-spectrum antibiotic are of importance for hospitals because of nosocomial resistant infections that have the potential of leading to lethal complications [44]. Consequently, a reduction in the usage of antibiotics can be utilized as a valid alternative to control or diminishing resistance [44].
Other drug usages also favor the growth of Candida species, especially $C$. albicans, are oral contraceptives, corticosteroids, and antiulcer drugs [44]. Corticosteroids mode of action is to promote anti-inflammatory and immunosuppressive properties; this can result in lower host resistance to infection and put the individuals at risk for candidiasis [50]. Corticosteroids often reduce the response of macrophages to T cell mediators [50]. Lastly, patients tend to have higher levels of salivary glucose; therefore, Candida growth, proliferation, and adhesion are promoted [70].

\section{Pregnancy, Neonates and Childhood}

Pregnancy can serve as a risk factor for vulvovaginal candidiasis [49]. Episodes of VVC and recurrent VVC often occur during pregnancy or the luteal phase of the menstruation cycle due to the elevated levels of estrogen and progesterone [71]. During pregnancy, the risk of developing VVC is increased due to the changes in hormone production which increases glycogen [49]. Pregnant women are commonly affected by C. albicans when suffering from VVC [49]. It has been shown in a study that pregnant women have an increased occurrence of vaginal Candida colonization compared to nonpregnant women [49].

Neonates Candida colonization results from vertical transmission from the infected mother [49,72]. Colonization may be the first step toward Candida infections at the rectumal in neonates [49]. In one study investigated what Candida isolate in neonates were similar to that of their mothers. The study determined that the mothers colonized with C. albicans give birth to neonates also colonized with C. albicans primarily in the anal region [72]. With the study's findings suggest that vertical transmission plays a vital role in neonates Candida colonization. Another study suggested that an increased risk of CCC occurs when the neonate's birth weight is below 1500 grams, received antibiotic treatment, and if extensive instruments were used during delivery [73].

Another risk for pregnant women and neonates is Candida chorioamnionitis. This infection is rare and characterized by inflammation of the amniochorion membrane; the membrane generally ruptures prematurely $[74,75]$. C. albicans $(71.3 \%$ of cases) is the most common species that has documented to cause this infection followed by C. glabrata [75]. One case study reported chorioamnionitis caused by C. glabrata followed by in vitro fertilization (IVF). This case study confirms that there is a strong association between fertilization techniques and chorioamnionitis [76]. More research is 
needed to deepen our knowledge about Candida infections and chorioamnionitis.

\section{Indwelling Medical Devices}

Approximately half of the nosocomial infections are associated with indwelling medical devices [28]. The consequence of having indwelling devices can lead to lifethreatening illness and device malfunction. A proportion of device-related infection is caused by Candida species; particularly the blood and urinary tract are involved. Also, biofilm formation insignificantly involved in these infections. The presence of a biofilm on the medical device poses another complication for treatment that was previously discussed as a virulence factor.

Common medical devices that have been associated with Candida infections include central venous catheters, cardiovascular devices, and urinary catheter. Most cases of urinary tract infections are catheters related, and Candida species accounted for approximately one-third of those infections [28]. Candida infections have a strong correlation with the presence of urinary catheters; $C$. albicans is the most commonly isolated species followed by C. glabrata [28].

Candida infections are also commonly associated with cardiovascular devices, including heart valves, pacemakers, implantable cardioverter defibrillators, and ventricular assist devices. Prosthetic valve endocarditis (PVE) is a rare but a severe complication; fungi have been reported to cause up to $10 \%$ of all cases of PVE and Candida species accounts for up to $90 \%$ of these infections [28]. Fungal PE has a higher mortality rate compared to bacterial PVE [28]. C. albicans is the most common species accounting for $56-66 \%$ of all Candida PVE; C. glabrata and C. parapsilosis have also been isolated $[77,78]$.

Another cardiovascular device that has been cited to have Candida infection complications are pacemakers; most often this complication is caused by Staphylococcus species [28]. Candida infections associated with pacemaker have been noted mostly as case studies or single case reports. Currently, cases have reported three Candida species as causative agents: C. albicans, C. glabrata, and, less often, C. tropicalis [79-81].

Implantable cardioverter defibrillators (ICD) are used to manage cardiac arrhythmias [28]. Infection rates for ICDs are low and have only been reported to reach about $7 \%$ [82]. One case study showed that of the seven infected patient, only one patient was infected with $C$. albicans and C. glabrata in conjunction with a bacterial infection [82].

\section{Virology \& Immunology Journal}

Therefore, fungal ICD infections are sporadic and commonly coincide with a bacterium.

Ventricular assist devices (VADs) provide temporary support for cardiac circulation [28]. Fungal colonization or infection have been documented in approximately 37 $\%$ of patients with VADs [83]. A recent study found that $38 \%$ of VAD patients developed Candidaemia $13.5 \%$ of all cases) [84]. Furthermore, VADs directly affects the host immune system; the interaction between the device and blood activates T cells resulting in cell death; also B cells become hyperactive with dysregulated immunoglobin synthesis [83]. These alternations in cellular immunity consequently increase the risk of fungal infections.

Central venous catheters are placed into large veins generally located on the neck, chest, or groin. Central venous catheter-related infection caused by fungi commonly manifests itself as fungemia [85]. Candidaemia is frequently among immunocompromised patients with underlying malignancy, hematologic disorders, GI diseases, burn, and critical illness [86]. C. albicans accounts for at least $60 \%$ of candidemia cases; C. glabrata and $C$. tropicalis have also been isolated in hospital settings [87]. One case report cited that nearly half of Candidaemia were caused by non-albicans species [88]. Another single case reported C. membranaefaciens as a causative agent [85]. This species is widely considered non-pathogenic and associated with drink spoilage.

\section{Recent or prolonged hospitalization}

Intensive care units (ICUs) have emerged as a hub for Candida infections. When admitted in the hospital, whether it be a short or long-term stay, one is at risk for a nosocomial infection. A cohort study was conducted over the course of 10 years of patients in ICUs. This study investigated the frequency of Candida infections in the context of IUCs. The most frequent infection was of the bloodstream (53\% of cases) and of those infections $C$. albicans was isolated 63.6\% [89]. Additionally, in this study, it noted other non- albicans species were also causative agents of Candidaemia, which included $C$. glabrata, C. tropicalis, C. parapsilosis, C.dubliniensis, and C. zeylanoides [89]. These infections were all related to hospital-borne infections. Therefore, it is vital that risk factors be followed up to prevent Candida based infections. This also brings up the controversy of if patients, who are in ICUs or going to have a prolonged stay at a healthcare facility, should be treated with antifungals as a prophylactic. 


\section{Clinical Manifestations}

\section{Oral Candidiasis}

Candida is readily isolated from the oral cavity, but this isolation is not a good predictive factor for the development of infection [90]. Oral candidiasis is commonly termed oral thrush; it frequently presents as a superficial infection. The oral manifestation of candidiasis is caused by the same species that commonly cause vaginal candidiasis. There is not one single form of oral candidiasis but rather three primary forms that are categorized according to their presentations.

\section{Pseudomembranous Candidiasis}

This manifestation of candidiasis is generally an acute infection, but if this infection is recurrent, then the term chronic pseudomembranous candidiasis is used. On the oral surfaces, a superficial layer of white to whitishyellow velvety confluent plaques that resemble cottage cheese is apparent; this layer can easily be slouched off [90-92]. The plaques consist of the accumulation of desquamated epithelial cells, yeast and hyphal form of Candida, keratin, fibrin, and necrotic cells [90-92]. Other surfaces that may be involved include labial and buccal mucosa, tongue, soft and hard palate, and oropharynx [90]. Patients with HIV/AIDS are prevalent to have both the oral and esophageal mucosa involved [90]. Individuals with the acute form report a mild tingling to a burning sensation and changes to taste perception $[90,92]$. Whereas patients with chronic pseudomembranous candidiasis the esophageal mucosa may be involved which then results in dysphagia and chest pains [90].

\section{Erythematous Candidiasis}

Erythematous candidiasis infections are not as standard; it manifests in an acute and chronic form $[90,92]$. Clinically, patients chief complaint is a localized painful sensation of the erythematous area [90]. Physical appearance includes lesions on the dorsum of the tongue with de-papillated areas [90]. Lesions may also appear on the palate; this is presented more common among HIV/AIDS patient s [90]. Erythematous candidiasis does not have a constant appearance; there are several variations if this oral candidiasis.

Acute atrophic candidiasis appears as red patches generally on the palate; these patches may also occur on the buccal mucosa or dorsal tongue [92]. Atrophy of the lingual papillae may accompany this infection [92]. Patients generally have complaints about a burning sensation of the mouth and soreness of the lips and tongue [92]. Clinically, this infection is generally localized within the erythematous regions [90]. Acute atrophic candidiasis is also known as antibiotic sore mouth

\section{Virology \& Immunology Journal}

because after prolonged use of broad-spectrum antimicrobials there is a possibility the overgrowth of $C$. albicans with the oral mucosa $[50,92]$.

Chronic atrophic candidiasis is also known as denture stomatitis; it is characterized by lesions that are erythematous, edematous, and infection is localized to the oral mucosa that in contact with dentures [92]. Generally, this infection is asymptomatic, but patients may have complaints of a burning sensation and or a sore mouth $[90,92]$. The infected area is typically restricted to the palate, but the mandibular mucosa may also be affected [90]. Studies have previously shown patients that wear dentures are at higher risk for oral colonization and or infection caused by C. glabrata [93].

Angular stomatitis physical appearance is redness and fissure lesions restricted to the commissures of the mouth, and often the fissures are bilateral. The primary complaint is a pain in the infected area. C. albicans is often isolated from the corners of the mouth in combination with Staphylococcus aureus and Streptococcus [90-92]. Although C. albicans is often isolated, cases have been reported of angular stomatitis being caused solely by bacteria along alone $[90,94]$.

Another form of oral candidiasis is median rhomboid glossitis. This infection causes symmetrical, red, and elliptical shaped lesions that are generally located on the posterior dorsal surface of the tongue [94]. Candida hyphae penetrate the superficial layers of the parakeratotic epithelium [94]. Also, the lesions are caused by the atrophy of the filiform papillae [92,94]; patients are ordinarily asymptomatic $[90,92,94]$. This manifestation is rare with a documented prevalence of less than $1 \%$ occurrence [92].

Lastly, linear gingival erythema was previously known as 'HIV-gingivitis' because it occurred in HIV in suffers and served as a clinical marker for the disease progression; although children have been reported with this condition [90,92]. Clinically, this infection manifests itself with erythematous bands on the marginal gingiva of one or more teeth; at times bleeding may occur [90,92]. Commonly C. albicans is associated with this infection, but C. dubliniensis has also been noted as an emerging pathogen $[90,91]$.

\section{Hyperplastic candidiasis}

Hyperplastic candidiasis previously was referred to as 'Candidal leukoplakia'; this was due to the difficulty to differentiate between leukoplakic and hyperplastic candidiasis [90,92]. Clinically, this Candida infection 


\section{Virology \& Immunology Journal}

presents itself in a chronic form [90]. Also, if clinically manifest itself in two variations: homogenous adherent plaque-like/smooth or as erythematous multiple nodular forms [90,91]. A primary characteristic of this form of candidiasis is the $C$. albicans hyphae penetrate the oral epithelium [91]. Lesions frequently form bilaterally in the commissural region of the buccal mucosa; the lesions less often form on the lateral border of the palate and tongue [90]. Hyperplastic candidiasis differs from pseudomembranous infections because the lesions from this form of infection cannot be gently removed by slouching sloughing [90-92]. Lacerations may be small to large translucent milky plaques, or they can be nodular or freckled upon looking atviewing [90-92]. Studies have shown aAnother important distinguishing feature of this candidiasis is studies have shown a possible connection with malignant transformation [91]. An established association between oral cancer and Candida has not been determined. However, in vitro studies have demonstrated that Candida organisms could generate carcinogenic nitrosamine $[63,91]$.

\section{Genitourinary Candidiasis in Males and Females}

Candida species are commonly found within the vaginal tract and preputial space $[95,96]$. The sole presence of Candida and the absence of immunosuppression and injured mucosa is not usually correlated; thus this is referenced as colonization [43]. The most frequent manifestation of genitourinary candidiasis is vulvovaginal candidiasis (VVC) followed by Candida balanitis and candiduria $[43,96,97]$. VVC is common among women in their childbearing years as well as patients who are on prolonged antibiotic therapy. The typical vaginal environment is maintained through the interactions of Lactobacillus acidophilus and resident flora, a hormonal and $\mathrm{pH}$ balance [12]. The vagina has a natural process in which it gains protection against reproductive tract infections; the lactobacilli breaks down into lactic acid creating an acidic environment that is toxic and inhibits the growth of other microorganisms including Candida species [12,98]. When this process experiences alterations to its equilibrium, such as antibiotic treatment, this can lead to vaginitis [12]. With this imbalance, Candida can over-proliferate; depending on the severity of this imbalance this infection can range from a mild yeast infection to disseminated infection in severely immunosuppressed patients $[12,43]$.

The healthy microflora of the preputial space is diverse, complex and changing as a result of type/presence of bacteria that is dependent on age, hygiene and sexual relations [95]. Similarly, to females, the simple presence of Candida species does not allow for a clinical diagnosis.

\section{Vulvovaginal Candidiasis (VVC) and Recurrent VVC}

VVC is characterized as the inflammation of the vulva and vaginal tract that is caused by Candida species $[12,43]$. Clinical symptoms of VVC vary among the cases and are nonspecific; also, the symptoms can be associated with other vaginal and sexual transmitted diseases [43]. Hallmark symptoms include itching, inflammation, burning, and thick curdy vaginal discharge with or without foul odor. C. albicans generally cause VVC albicans, but non-C. albicans species have been isolated in clinical cases as a causative agent, most commonly $C$. glabrata [96]. It has been widely assumed that the clinical presentation of VVC would be identical; however, subtle variances have been reported in clinical cases of $C$. glabrata VVC $[11,99]$. Women presenting symptoms of VVC caused by C. glabrata had a lower frequency of abnormal discharge when compared to C. albicans [11]. Patients also reported a burning sensation more regularly than itching [11]. In cases of VVC caused by C. glabrata, the patients were more likely to have elevated pH levels $[11,99]$. Since the frequency of vaginitis caused by $C$. glabrata is small, no large-scale studies have been conducted [11].

C. tropicalis is generally termed as the third most predominant Candida species, preceded by C. albicans and C. glabrata [100]. Vaginitis caused by $C$. tropicalis presents like a typical yeast infection. VVC infections caused by $C$. tropicalis is not commonly isolated in the United States and Europe regions but more commonly in the Nigeria regions [43]. Also, the patients' demographic effects and increase the probability of a NAC species infections.

Most VVC infections that are caused by non-albicans Candida are commonly caused by C. glabrata $(5 \%-10 \%$ of cases) or C. tropicalis (<5\% of cases) [101]. C. krusei has been reported being the causative agent of approximately $1 \%$ of vaginal candidiasis infections [102]. Vaginal candidiasis caused by $C$. krusei is unusual and questioned whether it is a true vaginal pathogen [101]. VVC caused by C. krusei appeared to have the same signs and symptoms from other vaginal candidiasis cases caused by other Candida species [102]. C. krusei is a rare causative agent for vaginal candidiasis but is of clinical importance due to its natural resistance to fluconazole $[101,102]$. Furthermore, there is limited research and literature experience with $C$. krusei vaginitis.

Candida parapsilosis is not frequently isolated from vaginal cultures, and its role as a VVC pathogen remains unstudied [103]. Women infected by $C$. parapsilosis reported signs and symptoms similar to a typical VVC infection caused by C. albicans [103]. 
Recurrent VVC is defined as having four or more vaginal yeast infections in a year [104]. Patients with recurrent VVC are predominantly infected by $C$. albicans or C. glabrata but determining the species is clinically essential for proper treatment and management [105]. It has been hypothesized that patients that have been experiencing recurrent VVC and are who are treated with short-term antifungals repeatedly may be the cause of the emergence of NAC vaginitis [106]. A higher percentage of NAC species have been clinically isolated from women with recurrent VVC [43]. Patients who present with unremarkable and challenging vaginitis yeast speciation is of importance. For example, patients who are infected with $C$. lusitaniae tend to report minimal symptomatology with less to no swelling or redness of the vaginal region; primarily report a burning or itching sensation [106]. Also, upon examination of these patients, the vaginal $\mathrm{pH}$ is healthy and have no external presentation of a yeast infection [106].

\section{Candida Balanitis}

Candida balanitis is associated with inflammation of the glans penis and regularly including the prepuce in the presence of Candida species $[43,95]$. Sole inflammation of the glans penis is referenced as balanitis, while inflammation of the prepuce is termed posthitis; if inflammation is involved in both areas, then it is coined balanoposthitis [95].

\section{Candiduria}

Candiduria is common among hospitalized patients but rare in healthy individuals [107]. Urinary tract infections (UTIs) caused by Candida species are on the rise mainly as nosocomial infectio ns due to the increase in at-risk patients $[107,108]$. Urine samples with the presence of Candida species pose a challenge for physicians in determining if the candiduria denotes colonization, urinary tract infection which has the possibility of leading to renal candidiasis with sepsis [108].

\section{Gastrointestinal candidiasis}

Candida species commonly colonize the human gastrointestinal tract as a commensal organism [109]. As an intestinal fungus is it believed that it directly influences gut health [110]. However, the direct influence of fungal colonization and its effects on the gastrointestinal tract has not been thoroughly studied. However new studies are coming out showing the results of an interaction between the intestinal immune system and the gut microbiome [111].

\section{Virology \& Immunology Journal}

\section{Candida colonization and GI tract diseases}

Inflammatory bowel disease (IBD) is a blanket term used to describe a heterogeneous group of chronic inflammatory diseases that involve the gastrointestinal (GI) tract $[109,111,112]$. IBD consist of two clinical manifestations: ulcerative colitis (UC) and Crohn's disease $[111,112]$.

Ulcerative colitis is defined by chronic continuous and superficial inflammation that affects the colon solely. A study was conducted to determine the effects of Candida colonization on the course of UC. The study yielded results that showed C. albicans (approx. 91\% of cases) was the predominant species followed by $C$. glabrata (approx. 6.7\% of cases) and C. inconspicua (approx. 1.6\% of cases) [112].

In comparison, Crohn's disease (CD) is characterized by patchy inflammation that may affect unspecified parts of the gastrointestinal tract and the entire thickness of the bowel wall. The specific cause of this disease is currently unknown but is hypothesized that there is an abnormal response to gut the microbial antigens $[111,113]$. One study observed the fungal changes of the gastrointestinal tract in the context of CD patients. From this study, it showed that patients diagnosed with $\mathrm{CD}$ had an increased abundance of C. tropicalis in correlation with Escherichia coli and Serratia marcescens [113]. Furthermore, there was also a high correlation with anti-saccharomyces cerevisiae antibodies (ASCA) titers in individuals that were heavily colonized $[113,114]$.

In conclusion, high levels of Candida colonization are associated with diseases of the gastrointestinal tract. The presence of Candida species, primarily C. albicans, is associated with delayed healing of gastric ulcers; also, inflammation and the ulceration aid in the colonization of the GI tract [109]. Future studies are needed to develop a more in-depth knowledge base of how these changes in the fungal population of the gastrointestinal tract result in IBD.

\section{Cutaneous Candidiasis}

\section{Congenital Cutaneous Candidiasis (CCC)}

Congenital cutaneous candidiasis (CCC) is an immensely rare disorder that first appears within the first week of life [73,115]. Neonates generally acquire candidiasis during the birthing process, but the reasons that explain the route of transmission and pathogenesis remains unknown [73]. It is currently being hypothesized that the development of chorioamnionitis is the mechanism of fetal infection [116]. CCC has a wide range of clinical manifestations $[73,115,116]$. Diagnosing CCC is 


\section{Virology \& Immunology Journal}

challenging due to the various rash presentations [117]. Congenital cutaneous candidiasis cases from 2004 and 2015 were reviewed and concluded that $100 \%$ of the cases C. albicans was isolated [117]. Generally, skin findings consist of an extensive maculopapular rash $[73,117]$. This neonate cutaneous infection manifestations also include erythema, pustules, abscesses, and or desquamation [73,115-117]. Typical areas of skin involvement are the face, scalp, trunk, and extremities [73,117]. CCC infections do have the ability to disseminate; generally, the patient presents with respiratory distress, and Candida species are isolated from the gastrointestinal tract; in rarer cases to the blood and cerebrospinal fluid [117].

Congenital cutaneous candidiasis is rare, and less than 100 cases have been reported in the literature over the past two decades. Therefore, little research and knowledge are known of this disorder.

\section{Chronic Mucocutaneous Candidiasis (CMC)}

Chronic mucocutaneous candidiasis is rare and characterized by recurrent Candida albicans infections of the skin, nails, mucous membranes and hyperkeratosis [118]. This disorder commonly manifests itself in early childhood, and there is some familial linkage. CMC disease is linked with inborn errors of the interleukin-17 (IL-17) immunity [118-120]. In 2011, two genetic etiologies were reported with this Candida disease: an autosomal recessive deficiency and an autosomal dominant deficiency [119]. Two separate cases of genetic deficiencies in genes for interleukin-17 signaling molecules have been noted [119]. The first patient presented an autosomal recessive deficiency of the interleukin-17 receptor A which eradicated the cellular responses downstream to IL-17A and IL-17F cytokines [119]. The second patient had an autosomal dominant deficiency in IL-17F cytokine resulted in a partial defect of the IL-17 dimer molecule [119]. Patients with an autosomal dominant deficiency commonly present with mutations of the signal transducer and activator of transcription 1 (STAT1) and caspase-associated recruitment domain (CARD9) [118,120]. STAT1 is a protein-coding gene that mediated cellular responses to signaling molec ules including interferons and cytokines. Mutations in STAT1 leads to defective helper-T (Th) cell 1 and 17 responses, which potentially explain this fungal infection $[118,120]$. CARD9 is an adaptor molecule that mediates signals from C-type lectin-like receptors (CLRs) to induce the transcription and production of proinflammatory cytokines [118,121]. Patients with CARD9 deficiencies present with two immunological phenotypes that include decreased IL-17 producing T cells and impaired C. albicans killing by neutrophils $[118,121]$.

\section{Candidal Onychomycosis}

Onychomycosis is a fungal infection of the nails; $80 \%$ of the toenail infections and at least $50 \%$ of fingernail infections are caused by dermatophytes [122,123]. However, Candida species have been emerging as a causative agent $[123,124]$. Candida species are not considered a part of the nail flora [44]. Candida onychomycosis is primarily found in individuals with defective immune responses consequentially due to aging, diabetes mellitus, and an immune suppressive disease. On the other hand, individuals that are not immunocompromised may also contract Candidal onychomycosis because of the persons' occupation. Candidal nail infections are more common among women than men who may be a result of self-inoculation from the vaginal flora [122,124]. Although some studies have shown that men are more likely to develop onychomycosis, then than women due to men increased lifestyle and occupation hazards [125]. This form of infection may manifest itself as paronychia and onychia $[44,122]$. Candida onychomycosis can be divided into three broad categories: Candidal paronychia, Candidal granuloma, and Candidal onycholysis [122,124].

\section{Candidal Paronychia}

This manifestation is the most common form of a Candidal nail infection and is characterized by initial inoculation of the soft tissue surrounding the nail which is then followed by the invasion of the nail plate $[122,124]$. The affected soft tissue is inflamed; generally appears red and edematous [124]. "Beau's lines," which are transverse depressions, commonly appear when the nail matrix is infected $[122,124]$. If the infection progresses this may result in a coarse, irregular, bowed, and finally dystrophic nail $[122,124]$. Paronychia presents either as an acute or chronic ailment [126]. The difference between these two presentations is the duration of the infection, acute paronychia is an infection that is last less than six weeks, and chronic is where the duration is longer than six weeks [126]. Candidal onychomycosis accounts for 95\% of chronic paronychia, and the remaining clinical cases are caused by atypical mycobacteria and gram-negative rods $[124,126]$. A present study showed that Candida parapsilosis and Candida guillermondii surpassed Candida albicans as the most commonly isolated pathogen from patients [127]. Within the same study, it showed the distribution of onychomycosis among Candida species might be dependent upon geographical location [127]. $C$. krusei, C. glabrata, C. tropicalis, and C. lusitaniae have also 
been isolated as causative agents for onychomycosis $[125,127]$.

\section{Candidal Granuloma}

Candidal granuloma exclusively appears in patients with CMC. The fungal organism directly invaded the nail plate, and when the infection is advanced, the soft tissue surrounding the nail is engorged until the digit develops a pseudo-clubbing appearance $[122,124]$.

\section{Candidal Onycholysis}

Onycholysis is defined as the separation of the nail plate from the nail bed that occurs on the finger and toes [128]. Initially, onycholysis appears at the distal subungual hyperkeratosis with a yellow to gray mass that lifts off from the nail plate [124]. As this infection progresses, the nail begins to lysis or separate $[122,124]$. This clinical manifestation is more common on the hands rather than the feet; also occurs mainly due to direct trauma to the nail of the fingers and toes $[122,124,128]$. Patients with this form commonly have psoriasis and or pustular psoriasis [122,124,128]. Either sex can be affected, but it is more common among women, potentially because of occupational hazard that allows the nails to be exposed to continuous moisture [128]. Finger onycholysis among women has been associated with $C$. albicans colonization [128].

\section{Invasive Candidiasis}

Clinical syndromes associated with invasive candidiasis caused by non-albicans species is difficult to differentiate from C. albicans. Manifestations range from fever in a hemodynamically stable patient to sepsis and septic shock [10]. Invasive candidiasis is a primary cause of disease and death in patients with hematologic malignancies and a frequent cause of failed remission induction chemotherapy [129]. At least 15 species of Candida causes diseases, but only about 5 of those are causative agents for invasive candidiasis: $C$. albicans, $C$. glabrata, C. tropicalis, C.krusei, and C. paraposilsis [130]. Also, in certain parts of the world, including Asia, the UK, Spain, and the United Staes, C. auris has been emerging as a pathogen. This is of concern because C. auris is a multidrug-resistant fungus [130].

Disseminated candidiasis can be either acute or chronic and commonly occurs in patients with leukemia who have received cytotoxic chemotherapy [129]. Clinically acute disseminated candidiasis (ADC) presents as neutropenic fever unresponsive to antibacterial, skin lesions, pneumonia, and shock. Common infection sites include the skin, lungs, GI tract, kidneys, liver, and spleen [129]. Chronic disseminated candidiasis (CDC) manifest

\section{Virology \& Immunology Journal}

as a non-neutropenic fever, unresponsive to antibacterial, abnormal liver function test; also, in this form of infection, the spleen and liver are predominantly the sites of infection [129].

Candidemia is generally viewed as the most common manifestation of candidiasis. Candidemia appears to be age-specific, with the highest occurrences at the extremes of age [131]. Deep-seated candidiasis commonly arises from direct inoculation or hematogenous dissemination. Common sites for deep-seated candidiasis include bone, muscle, joints, eyes, and the central nervous system (CNS) [131]. One possible way invasive candidiasis occurs when Candida species colonize the gut and invades through translocation to distal regions of the host. In patients with indwelling medical devices, candidemia that originated from colonization may lead to colonization of the device and the formation of a biofilm [131]. Once candidemia has been established, the fungi may disseminate to other regions. Signs and symptoms of candidemia are dependent upon the site of infection .

C. tropicalis is a frequent causative agent of candidemia in Latin America [132]. Patients who have this manifestation caused by $C$. tropicalis commonly central venous catheters and are on a broad-spectrum antibiotic [132]. Few reports of this infection exist, and the information is limited.

Also, C. glabrata has been reported as the cause of meningitis and endocarditis as a late complication of candidemia [133]. Candida infection of the CNS is a rare manifestation of invasive candidiasis. CNS infections caused by Candida species commonly includes meningitis, parenchymal abscesses, and vasculitis. Most cases are due to C. albicans [133].

\section{Diagnosis \& Therapeutic Treatment Options}

\section{Summary on Diagnosing Candidiasis}

Diagnosing Candida infections can be problematic because Candida species can grow in various morphological forms depending on their environment. Another challenge for diagnosing candidiasis is due to this infection presents itself in many manifestations; also, the manifestations may be dependent upon the patient's demographic. However, some tools can be applied to screen and determine the presence of fungal cells. Techniques include antigen testing, CHROMagar media, and microscopic examination [134].

Antigen testing for candidiasis is commercially available in enzyme-linked immunosorbent assay (ELISA) kits which are suitable for specificity but variable 


\section{Virology \& Immunology Journal}

sensitivity [134]. ELISA detects the mannans for the Candida species. Serum 1,3- $\beta$-D-glucan (BDG) is increasingly used as a diagnostic marker for invasive fungal infections [135]. Mannans and BDG are the most successful targets for these assays because they are the most abundant constituents of the cell wall. Serology testing is standard in diagnosing invasive candidiasis [136]. One primary concern with anti- Candida antibody assays is their sensitivity, which may be reduced in immunocompromised patients and a positive result may not differentiate previous infections [136].

CHROMagar is an agar plate that distinguishes between various Candida species by color because of a chemical reaction. This chromogenic media yields rapid identification of yeast. Currently, three Candida ( $C$. albicans, C. tropicalis, and C. krusei) species can be detected and differentiated. C. albicans growth is described as light-medium green colonies. C. tropicalis colonies are blue with a purple-like pigmentation in the surrounding agar. Lastly, C. krusei colony growths are large, fuzzy, and rose-like in color with white edges. Other Candida species can grow on this media and typically present as white-beige colonies (e.g., C. glabrata). Additionally, CHROMagar is commonly used to aid in identifying Candida involved in genitourinary infections [43]. This method of identification for Candida species is important because it is rapid and cost-effective.

Direct microscopic examination of smears is another approach for identifying Candida species. This approach is commonly used in Candida infections that involve the mucosal membranes (e.g., oral and genitourinary candidiasis) [43,137]. This method involves taking a sample from the infected area and viewing it under the microscope. Prior to examination, the sample is ideally treated with potassium hydroxide $(\mathrm{KOH})$, Gram stain, or periodic acid Schiff (PAS) stain [137]. Generally, when a Candida infection is suspected the samples are prepared with $\mathrm{KOH}$; $\mathrm{KOH}$ allows Candida to stand out and not be obscured by organic materials. Candida hyphae and pseudohyphae are visible in a $\mathrm{KOH}$ wet mount preparation [137]. Additionally, hyphae and yeast cells under Gram staining preparation will appear dark blue, whereas when in a PAS stain they will be red to purple [137].

\section{Summary of the Treatment of Candidiasis}

Currently, to date, there are three prin cipal antifungal agents to treat candidiasis which includes azoles, polyenes, and echinocandins. Each of these drug classes has a specific drug target.
Azoles target 14- $\alpha$-demethylase which mediates the conversion of lanosterol to ergosterol. This class directly targets the biosynthesis of ergosterol. Azoles are metabolized by cytochrome $\mathrm{p} 450$, which can cause a drug to drug interactions [138]. A common azole that is prescribed is Fluconazole. Fluconazole commonly treats candidemia and non-critically ill patients without prior exposure to azoles and colonization of the strain with reduced susceptibility to the azoles (e.g., VVC and candiduria) [138]. Fluconazole is generally well tolerated but has a limited spectrum; Voriconazole is the new azole on the market is the new and improved Fluconazole because of its increased spectrum.

Polyenes antifungal agents bind directly to ergosterol and destabilize the fungal cell membrane. Amphotericin $B$ $(\mathrm{AmB})$ is classified as polyene; $\mathrm{AmB}$ binds to the fungal cell membrane causing pores to form and subsequently the leakage of intracellular cations resulting in cell death. $\mathrm{AmB}$ is effective in killing fungal cells, but nephrotoxicity is a significant concern with this drug. Currently, there are three lipid formulations of AmB: liposomal amphotericin B (L-AmB), amphotericin B lipid complex (ABLC), and amphotericin $B$ colloidal dispersion (ABCD); all have a higher tolerance level and decrease toxicity [138]. Of the three lipid amphotericin B formulations, L-AmB is widely used and most favorable due to pharmacokinetics. L-AmB is also preferred because of its high intracellular penetration in the cerebral spinal fluid [138]. Commonly the first line of therapy for disseminated candidiasis is L$\mathrm{AmB}$ and used as the second line against invasive candidiasis [138].

Echinocandins directly targets the cell wall by inhibiting 1,3- $\beta$-D-glucan (BDG) synthesis; the loss of the cell wall glucans results in osmotic fragility. This is a fungicidal agent against Candida species including nonalbicans isolated resistance to Fluconazole. Echinocandins are generally well tolerated with limited side effects, also has excellent pharmacokinetics and pharmacodynamics. To date, three echinocandins (anidulafungin, caspofungin, and micafungin) are used as a treatment against invasive candidiasis, primarily candidemia [138]. Even though echinocandins have a strong safety profile, therapeutic concentrations are low in some tissues (e.g., eye, CNS, urine, endocardium, and peritoneum) also the pharmacodynamics and pharmacokinetics are poor in critically ill patients [138]. Echinocandins are concerned as a treatment option for invasive candidiasis in critically ill individuals and or when colonization of a Candida strain is evident with reduced susceptibility to azoles [138]. 


\section{Virology \& Immunology Journal}

5-fluorocytosine (Flucytosine) is another antifungal, and it inhibits nucleic acid synthesis. This therapy method is not commonly used as monotherapy because resistance easily develops. When is used flucytosine is used it is typically used in combination with another antifungal agent. Flucytosine use should be considered in cases of endophthalmitis, endocarditis or CNS infections because this drug distributes well among the tissues [138].

\section{Conclusion}

Candida species are an opportunistic fungal pathogen that causes infections ranging from superficial to invasive. The occurrences of opportunistic fungal infections have increased significantly over the past few decades, mainly contributes to morbidity and mortality in immunosuppressed patients. Although C. albicans may be the most prevalent species as of right now, the species distribution in the recent has begun to shift towards nonalbicans species. The NAC are emerging pathogens and are of clinical importance. These species have been largely ignored, and our current knowledge of pathogenicity, epidemiology, host immune response, and treatment of NAC infections is incomplete. Nevertheless, if NAC infections are to be adequately managed in the future comprehensive studies of these Candida species must be performed.

\section{References}

1. Sardi JCO, Scorzoni L, Bernardi T, Fusco-Almeida AM, Mendes Giannini MJ, et al. (2013) Candida species: current epidemiology, pathogenicity, biofilm formation, natural antifungal products and new therapeutic options. Journal of Medical Microbiology 62(1): 10-24.

2. Wise GJ, Talluri GS, VK Marella (1999) Fungal infections of the genitourinary system: manifestations, diagnosis, and treatment. Urologic Clinics of North America 26(4): 701-718.

3. Staib P, Morschhäuser J (2007) Chlamydospore formation in Candida albicans and Candida dubliniensis - an enigmatic developmental programme. Blackwell Publishing Ltd: Oxford, UK, pp: 1-12.

4. Thompson DS, Carlisle PL, Kadosh D (2011) Coevolution of Morphology and Virulence in Candida Species. Eukaryotic Cell 10(9): 1173-1182.
5. Chai LY, Denning DW, Warn P (2010) Candida tropicalis in human disease. Crit Rev Microbiol 36(4): 282-98.

6. Steffan P, Vazquez JA, Boikov D, Xu C, Sobel JD (1997) Identification of Candida species by randomly amplified polymorphic DNA fingerprinting of colony lysates. J Clin Microbiol 35(8): 2031-2039.

7. Yapar N (2014) Epidemiology and risk factors for invasive candidiasis. Therapeutics and Clinical Risk Management 10: 95-105.

8. Butler G, Rasmussen MD, Lin MF, Santos MA, Sakthikumar S, et al. (2009) Evolution of pathogenicity and sexual reproduction in eight Candida genomes. Nature 459(7247): 657-662.

9. Silva $S$, Negri $M$, Henriques $M$, Oliveira R, Williams DW, et al. (2012) Candida glabrata, Candida parapsilosis and Candida tropicalis: biology, epidemiology, pathogenicity and antifungal resistance. FEMS Microbiology Reviews 36(2): 288305.

10. Sobel JD (2006) The emergence of non-albicans Candida species as causes of invasive candidiasis and candidemia. Curr Infect Dis Rep 8(6): 427-343.

11. Fidel PL, Vazquez JA, Sobel JD (1999) Candida glabrata: Review of Epidemiology, Pathogenesis, and Clinical Disease with Comparison to C. albicans. Clinical Microbiology Reviews 12(1): 80-96.

12. Wariso K, IL Oboro, J Igunma (2007) Prevalence and clinical manifestations of candidiasis among patients with vulvovaginitis in a tertiary institution in Southern Nigeria.

13. Papon N, Courdavault V, Clastre M, Richard J, Bennett et al. (2013) Emerging and Emerged Pathogenic Candida Species: Beyond the Candida albicans Paradigm. PLOS Pathogens 9(9): e1003550.

14. Hernández-Chávez JM, Pérez-García LA, Niño-Vega GA, Mora-Montes HM (2017) Fungal Strategies to Evade the Host Immune Recognition. Journal of Fungi 3(4): 51 .

15. Ermert D Niemiec MJ, Röhm M, Glenthøj A, Borregaard N, et al. (2013) Candida albicans escapes from mouse neutrophils 94(2): 223-236.

16. Kaur R, Ma B, Cormack BP (2007) A family of glycosylphosphatidylinositol-linked aspartyl 
proteases is required for virulence of Candida glabrata. Proceedings of the National Academy of Sciences of the United States of America 104(18): 7628-7633.

17. Dementhon K, El-Kirat-Chatel S, Noël T (2012) Development of an In Vitro Model for the MultiParametric Quantification of the Cellular Interactions between Candida Yeasts and Phagocytes. PLOS ONE 7(3): e32621.

18. da Silva Dantas A, Lee KK, Raziunaite I, Schaefer K, Wagener J, et al. (2016) Cell biology of Candida albicans-host interactions. Current Opinion in Microbiology 34: 111-118.

19. Gantner BN, Simmons RM, Underhill DM (2005) Dectin-1 mediates macrophage recognition of Candida albicans yeast but not filaments. The EMBO Journal 24(6): 1277-1286.

20. Mayer FL, Wilson D, Hube B (2013) Candida albicans pathogenicity mechanisms. Virulence 4(2): 119-128.

21. Staab J, Bradway SD, Fidel PL, Sundstrom P (1999) Adhesive and mammalian transglutaminase substrate properties of Candida albicans Hwp 1. Science 283(5407): 1535-1538.

22. Brand A, Shanks S, Duncan VM, Yang M, Mackenzie K, et al. (2007) Hyphal Orientation of Candida albicans Is Regulated by a Calcium-Dependent Mechanism. Current Biology 17(4): 347-352.

23. Berman J (2012) Candida albicans. Current Biology 22(16): R620-R622.

24. Donlan RM (2002) Biofilms: Microbial Life on Surfaces. Emerging Infectious Diseases 8(9): 881-890.

25. Douglas LJ (2003) Candida biofilms and their role in infection. Trends in Microbiology 11(1): 30-36.

26. Nobile CJ, Johnson AD (2015) Candida albicans Biofilms and Human Disease. Annual review of microbiology 69: 71-92.

27. Ramage G, Bachmann S, Patterson TF, Brian L, Wickes, et al. (2002) Investigation of multidrug efflux pumps in relation to fluconazole resistance in Candida albicans biofilms. Journal of Antimicrobial Chemotherapy 49(6): 973-980.

\section{Virology \& Immunology Journal}

28. Kojic EM, Darouiche RO (2004) Candida Infections of Medical Devices. Clinical Microbiology Reviews 17(2): 255-267.

29. Hawser SP, LJ Douglas (1994) Biofilm formation by Candida species on the surface of catheter materials in vitro. Infection and Immunity 62(3): 915-921.

30. Li X, Yan Z, Xu J (2003) Quantitative variation of biofilms among strains in natural populations of Candida albicans. Microbiology 149(2): 353-362.

31. Kuhn DM, Chandra J, Mukherjee PK, Ghannoum MA (2002) Comparison of Biofilms Formed by Candida albicans and Candida parapsilosis on Bioprosthetic Surfaces. Infection and Immunity 70(2): 878-888.

32. Cheng SC, van de Veerdonk F, Smeekens S, Joosten LA, van der Meer JW, et al. (2010) Candida albicans dampens host defense by downregulating IL-17 production. Journal of immunology 185(4): 24502457.

33. Cheng SC, Joosten LA, Kullberg BJ, Netea MG (2012) Interplay between Candida albicans and the Mammalian Innate Host Defense. Infection and Immunity 80(4): 1304-1313.

34. Dumitru R, Navarathna DH, Semighini CP, Elowsky CG, Dumitru RV, et al. (2007) In Vivo and In Vitro Anaerobic Mating in Candida albicans. Eukaryotic Cell 6(3): 465-472.

35. Miyazato A, Nakamura K, Yamamoto N, Mora Montes HM, Tanaka M, et al. (2009) Toll-Like Receptor 9Dependent Activation of Myeloid Dendritic Cells by Deoxynucleic Acids from Candida albicans. Infection and Immunity 77(7): 3056-3064.

36. Kasperkovitz PV, Khan NS, Tam JM, Mansour MK, Davids PJ, et al. (2011) Toll-like receptor 9 modulates macrophage antifungal effector function during innate recognition of Candida albicans and Saccharomyces cerevisiae. Infection and Immunity 79(12): 4858-4867.

37. Chen SM, Shen H, Zhang T, Huang X, Liu XQ, et al. (2017) Dectin-1 plays an important role in host defense against systemic Candida glabrata infection. Virulence 8(8): 1643-1656.

38. Kim YK, Shin JS, Nahm MH (2016) NOD-Like Receptors in Infection, Immunity, and Diseases. Yonsei Medical Journal 57(1): 5-14. 
39. Zheng NX, Wang Y, Hu DD, Yan L, Jiang YY (2015) The role of pattern recognition receptors in the innate recognition of Candida albicans. Virulence 6(4): 347 361.

40. Joly S, Eisenbarth SC, Olivier AK, Williams A, Kaplan DH, et al. (2012) Nlrp10 is essential for protective anti-fungal adaptive immunity against Candida albicans. Journal of immunology 189(10): 4713-4717.

41. Tomalka J, Ganesan S, Azodi E, Patel K, Majmudar P, et al. (2011) A Novel Role for the NLRC4 Inflammasome in Mucosal Defenses against the Fungal Pathogen Candida albicans. PLoS Pathogens 7(12): e1002379.

42. Richards MJ, Edwards JR, Culver DH, Gaynes RP (2000) Nosocomial Infections in Combined MedicalSurgical Intensive Care Units in the United States. Infection Control Epidemiology 21(8): 510-515.

43. Achkar JM, Fries BC (2010) Candida Infections of the Genitourinary Tract. Clinical Microbiology Reviews 23(2): 253-273.

44. Martins N, Ferreira IC, Barros L, Silva S, Henriques M (2014) Candidiasis: Predisposing Factors, Prevention, Diagnosis and Alternative Treatment. Mycopathologia 177(5): 223-240.

45. Pfaller MA, Diekema DJ (2007) Epidemiology of Invasive Candidiasis: a Persistent Public Health Problem. Clinical Microbiology Reviews 20(1): 133163.

46. Paillaud E, Merlier I, Dupeyron C, Scherman E, Poupon J, et al. (2004) Oral candidiasis and nutritional deficiencies in elderly hospitalised patients. British Journal of Nutrition 92(5): 861-867.

47. Paš M, Piskur B, Sustaric M, Raspor P (2007) Iron enriched yeast biomass-A promising mineral feed supplement. Bioresource Technology 98(8): 1622 1628.

48. Serrano R, Bernal D, Simón E, Ariño J (2004) Copper and Iron Are the Limiting Factors for Growth of the Yeast Saccharomyces cerevisiae in an Alkaline Environment. Journal of Biological Chemistry 279(19): 19698-19704.

49. Singh S, Fatima Z, Hameed S (2015) Predisposing factors endorsing Candida infections. Infez Med 23(3): 211-223.

\section{Virology \& Immunology Journal}

50. Van Wyk C, Steenkamp V (2011) Host factors affecting oral candidiasis. Southern African Journal of Epidemiology and Infection 26(1): 18-21.

51. de Leon EM, Jacober SJ, Sobel JD, Foxman B (2002) Prevalence and risk factors for vaginal Candidacolonization in women with type 1 and type 2 diabetes. BMC Infectious Diseases 2(1): 1.

52. Pallavan B, Ramesh V, Dhanasekaran BP, Oza N, Indu $S$, et al. (2014) Comparison and correlation of Candidal colonization in diabetic patients and normal individuals. Journal of Diabetes and Metabolic Disorders 13: 66.

53. Kwamin F, Nii Otu N, Francis SC, Mercy J (2013) Newman Distribution of Candida species among HIVpositive patients with oropharyngeal candidiasis in Accra, Ghana. Journal of Infection in Developing Countries 7(1): 41-45.

54. Rodriguez Rodrigues C, Remes Lenicov F, Jancic C, Sabatté J, Cabrini M, et al. (2013) Candida albicans Delays HIV-1 Replication in Macrophages. PLoS ONE 8(8): e72814.

55. Das PP, Saikia L, Nath R, Phukan SK (2016) Species distribution \& antifungal susceptibility pattern of oropharyngeal Candida isolates from human immunodeficiency virus infected individuals. Indian Journal of Medical Research 143(4): 495-501.

56. Muhammad T, Ibrahim M Hamidu, Idris Abdullahi Nasir, Abubakar Mursal, Kalama B Dikwa, et al. (2017)Prevalence and profile of pulmonary fungal pathogens among HIV-infected patients attending University of Maiduguri Teaching Hospital, Nigeria. The Egyptian Journal of Internal Medicine 29(1): 1115.

57. Maurya V, Srivastava A, Mishra J, Gaind R, Marak RS, et al. (2013) Oropharyngeal candidiasis and Candida colonization in HIV positive patients in northern India. Journal of Infection in Developing Countries $7(8):$ 608-613.

58. Cassone A, Cauda R (2012) Candida and candidiasis in HIV-infected patients: where commensalism, opportunistic behavior and frank pathogenicity lose their borders. AIDS 26(12): 1457-1472.

59. Koziol Montewka M, Magrys A, Paluch Oles J, Bogut A, Buczynski K, et al. (2006) MPO and Cytokines in the Serum of Cancer Patients in the Context of Candida 
Colonization and Infection. Immunological Investigations 35(2): 167-179.

60. Martin Manso G, Susana Galli, David R Soto Pantoja, Svetlana A Kuznetsova, Maria Tsokos, et al. (2012) Endogenous Thrombospondin-1 Regulates Leukocyte Recruitment and Activation and Accelerates Death from Systemic Candidiasis. PLOS ONE 7(11): e48775.

61. Chung LM, Liang JA, Lin CL4, Sun LM, Kao CH, et al. (2017) Cancer risk in patients with candidiasis: a nationwide population-based cohort study. Oncotarget 8(38): 63562-63573.

62. Ramirez-Garcia A, Rementeria A, Aguirre-Urizar JM, Moragues MD, Antoran A, et al. (2016) Candida albicans and cancer: Can this yeast induce cancer development or progression? Crit Rev Microbiol 42(2): 181-193.

63. Krogh P, Hald B, Holmstrup P (1987) Possible mycological etiology of oral cancer: Catalytic potential of infecting Candida albicans and other yeasts in production of N-nitrosobenzyl-methylamine. Carcinogenesis 8(10): 1543-1548.

64. Krogh P, Holmstrup P, Thorn JJ, Vedtofte P, Pindborg JJ, et al. (1987) Yeast species and biotypes associated with oral leukoplakia and lichen planus. Oral Surgery, Oral Medicine, Oral Pathology 63(1): 48-54.

65. O'Grady JF, Reade PC (1992) Candida albicans as a promoter of oral mucosal neoplasia. Carcinogenesis 13(5): 783-786.

66. Marttila E, Bowyer P, Sanglard D, Uittamo J, Kaihovaara P, et al. (2013) Fermentative 2-carbon metabolism produces carcinogenic levels of acetaldehyde in C andida albicans. Molecular Oral Microbiology 28(4): 281-291.

67. Ramirez-Garcia A, Beatriz Arteta, Ana Abad-Diaz-deCerio, Aize Pellon, Aitziber Antoran, et al. (2013) Candida albicans Increases Tumor Cell Adhesion to Endothelial Cells In Vitro: Intraspecific Differences and Importance of the Mannose Receptor. Plos One 8(1): 53584.

68. Rodríguez-Cuesta J, Hernando FL, Mendoza L, Gallot $\mathrm{N}$, de Cerio AA, et al. (2010) Candida albicans enhances experimental hepatic melanoma metastasis. Clinical \& Experimental Metastasis 27(1): 35-42.

\section{Virology \& Immunology Journal}

69. Langowski JL, Kastelein RA, Oft M (2007) Swords into plowshares: IL-23 repurposes tumor immune surveillance. Trends in Immunology 28(5): 207-212.

70. Knight L, Fletcher J (1971) Growth of Candida albicans in Saliva: Stimulation by Glucose Associated with Antibiotics, Corticosteroids, and Diabetes Mellitus. The Journal of Infectious Diseases 123(4): 371-377.

71. Paul L. Fidel, Jessica Cutright, Chad Steele (2000) Effects of Reproductive Hormones on Experimental Vaginal Candidiasis. Infection and Immunity 68(2): 651-657.

72. Filippidi A, Galanakis E, Maraki S, Galani I, DrogariApiranthitou M, et al. (2014) The effect of maternal flora on $\mathrm{C}$ andida colonisation in the neonate. Mycoses 57(1): 43-48.

73. Sujit A Jagtap, Pallavi P Saple, Saleha B Dhaliat (2011) Congenital cutaneous candidiasis: $A$ rare and unpredictable disease. Indian Journal of Dermatology 56(1): 92-93.

74. Czikk MJ, McCarthy FP, Murphy KE (2011) Chorioamnionitis: from pathogenesis to treatment. Clinical Microbiology and Infection 17(9): 1304-1311.

75. Yohei Maki, Midori Fujisaki, Yuichiro Sato, Hiroshi Sameshima (2017) Candida Chorioamnionitis Leads to Preterm Birth and Adverse Fetal-Neonatal Outcome. Infectious Diseases in Obstetrics and Gynecology, pp: 1-11.

76. Jackel D, Lai K (2013) Candida glabrata sepsis associated with chorioamnionitis in an in vitro fertilization pregnancy: case report and review. Clinical infectious diseases 56(4): 555-558.

77. Rana M Nasser, Gerardo R Melgar, David L Longworth, Steven M Gordon (1997) Incidence and Risk of Developing Fungal Prosthetic Valve Endocarditis after Nosocomial Candidemia. The American Journal of Medicine 103(1): 25-32.

78. Melgar GR, Nasser RM, Gordon SM, Lytle BW, Keys TF, et al. (1997) Fungal Prosthetic Valve Endocarditis in 16 Patients An 11-Year Experience in a Tertiary Care Hospital. Medicine 76(2): 94-103.

79. Rogera PM, Boissy C, Gari-Toussaintc M, Foucherd R, Mondain V, et al. (200) Medical Treatment of a Pacemaker Endocarditis due toCandida albicansand 


\section{Virology \& Immunology Journal}

to Candida glabrata. Journal of Infection 41(2): 176178.

80. Kurup A, Janardhan MN, Seng TY (2000) Candida tropicalis Pacemaker Endocarditis. Journal of Infection 41(3): 275-276.

81. Joly V, Belmatoug N, Leperre A, Robert J, Jault F, et al. (1997) Pacemaker endocarditis due to Candida albicans: Case report and review. Clin Infect Dis 25(6): 1359-1362.

82. Kwan Kew L, Sally AF (1998) Infections Associated with Implantable Cardioverter Defibrillators Placed Transvenously and via Thoracotomies: Epidemiology, Infection Control, and Management. Clinical Infectious Diseases 27(2): 265-269.

83. Goldberg Steven P, Baddley John W, Aaron Mark F, Pappas Peter G, Holman William L, et al. (2000) Fungal Infections in Ventricular Assist Devices. ASAIO Journal 46(6): 37-40.

84. Gordon SM, Steven K Schmitt, Micah Jacobs BS, Nicolas M Smedira, Marlene Goormastic, et al. (2001) Nosocomial bloodstream infections in patients with implantable left ventricular assist devices. The Annals of Thoracic Surgery 72(3): 725-730.

85. Fanci R, Pecile P (2005) Central venous catheter-related infection due to Candida membranaefaciens , a new opportunistic azole-resistant yeast in a cancer patient: a case report and a review of literature. Mycoses 48(5): 357-359.

86. Wey SB, Mori M, Pfaller MA, Woolson RF, Wenzel RP, et al. (1989) Risk factors for hospital-acquired candidemia: A matched case-control study. Archives of Internal Medicine 149(10): 2349-2353.

87. Fraser VJ, Jones M, Dunkel J, Storfer S, Medoff G, et al. (1992) Candidemia in a tertiary care hospital: epidemiology, risk factors, and predictors of mortality. Clin Infect Dis 15(3): 414-421.

88. Nguyen MH, Peacock JE, Morris AJ, Tanner DC, Nguyen ML, et al. (1996) The changing face of candidemia: emergence of non-Candida albicans species and antifungal resistance. The American Journal of Medicine 100(6): 617-623.

89. Zehra Karacaer, Oral Oncul, Vedat Turhan, Levent Gorenek, Mustafa Ozyurt, et al. (2014) A surveillance of nosocomial Candida infections: epidemiology and influences on mortalty in intensive care units. The Pan African Medical Journal 19: 398.

90. Shankargouda Patil, Roopa S Rao, Barnali Majumdar, Sukumaran Anil (2015) Clinical Appearance of Oral Candida Infection and Therapeutic Strategies. Frontiers in Microbiology 6: 1391.

91. Williams D, Lewis M (2011) Pathogenesis and treatment of oral candidosis. J Oral Microbiol, pp: 3.

92. Millsop JW, Fazel N (2016) Oral candidiasis. Clinics in Dermatology 34(4): 487-494.

93. Li L, Redding S, Dongari-Bagtzoglou A (2007) Candida glabrata an Emerging Oral Opportunistic Pathogen. Journal of Dental Research 86(3): 204-215.

94. Farah C, Lynch N, McCullough M (2010) Oral fungal infections: an update for the general practitioner. Aust Dent J 55: 48-54.

95. Aridogan IA, Izol V, Ilkit M (2011) Superficial fungal infections of the male genitalia: A review. Critical Reviews in Microbiology 37(3): 237-244.

96. White DJ, Vanthuyne A (2006) Vulvovaginal candidiasis. Sexually Transmitted Infections 82(S4): 428-430.

97. Sobel JD, Faro S, Force RW, Foxman B, Ledger WJ, et al. (1998) Vulvovaginal candidiasis: Epidemiologic, diagnostic, and therapeutic considerations. Am J Obstet Gynecol 178(2): 203-211.

98. O'Hanlon DE, Moench TR, Cone RA (2013) vaginal pH and Microbicidal Lactic Acid When Lactobacilli Dominate the Microbiota. PLoS One 8(11).

99. Geiger AM, Foxman B, Sobel JD (1995) Chronic vulvovaginal candidiasis: characteristics of women with Candida albicans, C glabrata and no Candida. Genitourinary Medicine 71(5): 304-307.

100. Zuza-Alves DL, Silva-Rocha WP, Chaves GM (2017) An Update on Candida tropicalis Based on Basic and Clinical Approaches. Front Microbiol 8(1927).

101. Singh S, Sobel JD, Bhargava P, Boikov D, Vazquez JA (2002) Vaginitis Due to Candida krusei: Epidemiology, Clinical Aspects, and Therapy. Clin Infect Dis 35(9): 1066-1070.

102. Guzel AB, Aydın M, Meral M, Kalkancı A, Ilkit M (2013) Clinical Characteristics of Turkish Women 
with Candida krusei Vaginitis and Antifungal Susceptibility of the C. krusei Isolates. Infect Dis Obstet Gynecol 2013: 698736.

103. Nyirjesy P, Alexander AB, Weitz MV (2005) Vaginal Candida parapsilosis: pathogen or bystander? Infect Dis Obstet Gynecol 13(1): 37-41.

104. Bernstein JA, Seidu L (2015) Chronic vulvovaginal Candida hypersensitivity: An underrecognized and undertreated disorder by allergists. Allergy \& Rhinology 6(1): e44-e49.

105. Sobel JD (2016) Recurrent vulvovaginal candidiasis. American Journal of Obstetrics and Gynecology 214(1): 15-21.

106. Silverman NS, Morgan M, Nichols WS (2001) Candida lusitaniae as an Unusual Cause of Recurrent Vaginitis and its Successful Treatment With Intravaginal Boric Acid. Infect Dis Obstet Gynecol 9(4): 245-247.

107. Sobel JD, Kauffman CA, McKinsey D, Zervos M, Vazquez JA, et al. (2000) Candiduria: A Randomized, Double-Blind Study of Treatment with Fluconazole and Placebo. Clinical Infectious Diseases 30(1): 1924.

108. Bukhary Z (2008) Candiduria: A Review of Clinical Significance and Management. Saudi Journal of Kidney Dis Transplant 19(3): 350-360.

109. Kumamoto CA (2011) Inflammation and gastrointestinal Candida colonization. Curr opin microbiol 14(4): 386-391.

110. Qiu X, Zhang F, Yang X, Wu N, Jiang W, et al. (2015) Changes in the composition of intestinal fungi and their role in mice with dextran sulfate sodiuminduced colitis. Scientific Reports 5: 10416.

111. Lane ER, Zisman TL, Suskind DL (2017) The microbiota in inflammatory bowel disease: current and therapeutic insights. J Inflamm Res 10: 63-73.

112. Zwolinska-Wcislo M, Brzozowski T, Budak A, Kwiecien S, Sliwowski Z, et al. (2009) Effect of Candida colonization on human ulcerative colitis and the healing of inflammatory changes of the colon in the experimental model of Colitis ulcerosa. J Physiol Pharmacol 60(1): 107-118.

113. Hoarau G, Mukherjee PK, Gower-Rousseau C, Hager C, Chandra J, et al. (2016) Bacteriome and

\section{Virology \& Immunology Journal}

Mycobiome Interactions Underscore Microbial Dysbiosis in Familial Crohn's Disease. MBio 7(5).

114. Sutton CL, Yang H, Li Z, Rotter JI, Targan SR, et al. (2000) Familial expression of anti-Saccharomyces cerevisiae mannan antibodies in affected and unaffected relatives of patients with Crohn's disease. Gut 46(1): 58-63.

115. Darmstadt GL, Dinulos JG, Miller Z (200) Congenital Cutaneous Candidiasis: Clinical Presentation, Pathogenesis, and Management Guidelines. Pediatrics 105(2): 438-444.

116. Aldana-Valenzuela C, Morales-Marquec M, Castellanos-Martinez J, Deanda-Gomez M (2005) Congenital Candidiasis: A Rare and Unpredictable Disease. J Perinatol 25(10): 680-682.

117. Kaufman DA, Coggins SA, Zanelli SA, Weitkamp JH (2017) Congenital Cutaneous Candidiasis: Prompt Systemic Treatment Is Associated With Improved Outcomes in Neonates. Clinical Infectious Diseases 64(10): 1387-1395.

118. Okada S, Puel A, Casanova JL, Kobayashi M (2016) Chronic mucocutaneous candidiasis disease associated with inborn errors of IL-17 immunity. Clin Trans Immunol 5(12): e114.

119. Puel A, Cypowyj S, Bustamante J, Wright JF, Liu L, et al. (2011) Chronic Mucocutaneous Candidiasis in Humans with Inborn Errors of Interleukin-17 Immunity. Science 332(6025): 65-68.

120. van de Veerdonk FL, Plantinga TS, Hoischen A, Smeekens SP, Joosten LA, et al. (2011) STAT1 Mutations in Autosomal Dominant Chronic Mucocutaneous Candidiasis. N Eng J Med 365(1): 5461.

121. Drummond RA, Lionakis MS (2016) Mechanistic Insights into the Role of C-Type Lectin Receptor/CARD9 Signaling in Human Antifungal Immunity. Frontiers in Cellular and Infection Microbiology 6: 39.

122. Elewski BE (1998) Onychomycosis: Pathogenesis, Diagnosis, and Management. Clinical Microbiology Reviews 11(3): 415-429.

123. Shirwaikar AA, Thomas T, Shirwaikar A, Lobo R, Prabhu KS (2008) Treatment of onychomycosis: An update. Indian Journal of Pharmaceutical Sciences 70(6): 710-714. 


\section{Virology \& Immunology Journal}

124. Jayatilake JA, Tilakaratne WM, Panagoda GJ (2009) Candidal onychomycosis: a mini-review. Mycopathologia 168(4): 165-173.

125. Ghannoum MA, Hajjeh RA, Scher R, Konnikov N, Gupta AK, et al. (2000) A large-scale North American study of fungal isolates from nails: The frequency of onychomycosis, fungal distribution, and antifungal susceptibility patterns. Journal of the American Academy of Dermatology 43(4): 641-648.

126. Rockwell PG (2001) Acute and chronic paronychia. Am Fam Physician 63(6): 1113-1116.

127. Fich F, Abarzúa-Araya A, Pérez M, Nauhm Y, León E (2014) Candida Parapsilosis and Candida Guillermondii: Emerging Pathogens in Nail Candidiasis. Indian Journal of Dermatology 59(1): 24-29.

128. Zaias N, Escovar SX, Zaiac MN (2015) Finger and toenail onycholysis. Journal of the European Academy of Dermatology and Venereology 29(5): 848-853.

129. Kontoyiannis DP, Luna MA, Samuels BI, Bodey GP (2000) HEPATOSPLENIC CANDIDIASIS: A Manifestation of Chronic Disseminated Candidiasis. Infectious Disease Clinics of North America 14(3): 721-739.

130. Pappas PG, Lionakis MS, Arendrup MC, OstroskyZeichner L, Kullberg BJ (2018) Invasive candidiasis. Nature Reviews Disease Primers 4: 18026.
131. Kullberg BJ, Arendrup MC (2015) Invasive Candidiasis. New England Journal of Medicine 373(15): 1445-1456.

132. Goldani LZ, Mário PS (2003) Candida tropicalis Fungemia in a Tertiary Care Hospital. Journal of Infection 46(3): 155-160.

133. Colomba, C., Trizzino M, Imburgia C, Madonia S, Siracusa L, et al. (2014) Candida glabrata meningitis and endocarditis: a late severe complication of candidemia. International Journal of Infectious Diseases 29: 174-175.

134. Barnes RA (2008) Early diagnosis of fungal infection in immunocompromised patients. Journal of Antimicrobial Chemotherapy 61(S1): i3-i6.

135. Shupp JW, Petraitiene R, Jaskille AD, Pavlovich AR, Matt SE, et al. (2012) Early serum (1 $\rightarrow 3)-\beta$-D-glucan levels in patients with burn injury. Mycoses 55(3): 224-227.

136. Clancy CJ, Nguyen MH (2018) Diagnosing Invasive Candidiasis. Journal of clinical microbiology 56(5): e01909-17.

137. Marty M, Bourrat E, Vaysse F, Bonner M, BailleulForestier I (2015) Direct Microscopy: A Useful Tool to Diagnose Oral Candidiasis in Children and Adolescents. Mycopathologia 180(5): 373-377.

138. Bassetti M, Peghin M, Timsit JF (2016) The current treatment landscape: candidiasis. Journal of Antimicrobial Chemotherapy 71(suppl_2): ii13-ii22. 